\title{
Creativity Support and Multimodal Pen-based Interaction
}

\author{
Leave Authors Anonymous \\ for Submission \\ City, Country \\ e-mail address
}

\author{
Leave Authors Anonymous \\ for Submission \\ City, Country \\ e-mail address
}

\author{
Leave Authors Anonymous \\ for Submission \\ City, Country \\ e-mail address
}

\begin{abstract}
Creativity as a skill is associated with a potential to drive both productivity and psychological wellbeing. Since multimodality can foster cognitive ability, multimodal digital tools should also be ideal to support creativity as an essentially cognitive skill. In this paper, we explore this notion by presenting a multimodal pen-based interaction technique and studying how it supports creativity. The multimodal solution uses microcontroller-technology to augment a digital pen with RGB LEDs and a Leap Motion sensor to enable bimanual input. We report on a user study with 26 participants demonstrating that the multimodal technique is indeed perceived as supporting creativity significantly more than a baseline condition. We conclude with a critical discussion of our results, considering implications for creativity support through multimodal interaction techniques and the culture and materiality surrounding lived practices of pen-based sketching. To this end, we utilize insights based on our own experience observing and engaging with various sketching communities in our town, including the urban sketchers.
\end{abstract}

\section{ACM Classification Keywords}

H.5.m. Information Interfacess and Presentation (e.g. HCI): Miscellaneous;

\section{Author Keywords}

Multimodality; creativity support, pen input; mid-air input; embedded technology; interaction design.

\section{INTRODUCTION}

The role of technology for people of all walks of life, young and old has been increasing over the last few decades, with technology taking an increasingly important part in how people live, learn, and feel. Consequently, the diversity of user experiences and interaction techniques that are studied and supported by multimodal interaction design has also broadened. One could argue that multimodal interfaces initially focussed on improving traditional usability by, for example distributing cognitive resources on multiple modalities [44] and consequently allowing tasks to be solved faster or more

submitted to ICMI'19, todo

ACM ISBN .

DOI: accurately and with less error. In addition to potentially improving such pragmatic interface qualities, recent work in multimodal interaction techniques has also demonstrated that multimodality can result in improvements in an interface's perceived hedonic qualities and increase for example attractiveness of an interaction technique or a product (e.g., [1]). Thus, in a nutshell, we as a research community are aware that multimodality has the potential to improve the productivity and attractiveness of existing applications or tools.

But there are more qualities that interaction designers of multimodal interfaces are increasingly becoming aware of and that they should include in their repertoire of design goals. Calvo and Peters [13] mention human potential and flourishing as important factors, which could potentially have a positive longterm impact on psychological wellbeing, and thus, differ from what typical hedonic experience designs aim for (e.g., shortterm "wow effects" to sell more products). Moreover, they argue that human flourishing can be achieved by designing for (or supporting) well understood psychological wellbeing determinants, such as autonomy, competence, and mindfulness.

At about the same time that Calvo and Peters criticized design goals for technology and their application, Sharon Oviatt highlighted in her keynote at ICMI 2014 that multimodal interaction research could focus on tools and interaction techniques, which may stimulate thought and cognitive ability in a broader sense. She provided as a paradigmatic example the historical impact of traditional pens and how compared to digital pens, mouse and keyboard-based interaction may "limit" cognitive ability and should therefore, especially in learning domains, be less prioritized.

The research at hand is motivated to bring together research threads in multimodal interaction and creativity support. We do this because we believe that creativity support is an increasingly valuable attribute of a digital tool with a potential impact on both productivity and psychological wellbeing. Despite the fact that multimodal solutions may have always supported creativity by providing alternative interaction techniques, increasing expressivity of interaction, and freeing cognitive resources, to our great surprise, there seems to exist a gap in multimodal interaction research explicitly addressing creativity support.

Towards closing this gab, we first present a multimodal pen prototype, which we hypothesis will support creativity more than a baseline version. Then we report on a user study with 26 participants, which clearly demonstrates that the multimodal version supports creativity significantly more than the baseline condition. In order to shed light into possible practical 
implications, we discuss the study results by considering creativity support practices of sketching communities in our own town (i.e., nude painters and urban sketchers), including how these communities feel about technology usage. Before we provide the multimodal pen prototype and report the results of our study in detail we provide in the next section background, including related multimodal interaction techniques and methods to measure creativity support.

\section{BACKGROUND}

\section{Creativity Support}

Creativity is a skill that has significance in various fields of application. For companies, creativity, along with innovation, design and speed, is an important property for developing and selling products [31]. In addition, creativity provides a personal benefit for users. As versatile as the application are the tools available to foster creativity. The tools can be divided into different classes to promote the creativity of individuals and groups: e.g., simulation, animation and interaction, visualization tools, music, concept mapping, and video editing [41]. From creativity tools for songwriters [40], interactive tools for designers [46], applications to promote business innovation [29] or suggestions to encourage creativity by scientists [19] are just a few examples of how tools can be used to foster creativity. Another tool that can be seen as the basis of creative activity is the pen. With this production tool, people are able to implement creative ideas without great effort. In addition to the use on materials such as paper or canvas, which has been used for centuries, pens can also be used digitally with the development of tablets and smart pads.

\section{Creativity Measurement}

In addition to the use of various tools to express and improve creativity, an important focus lays on the measurement of creativity. These instruments allow to investigate the change of various creativity aspects of the user (see Table 1). They are also useful to measure the effectiveness of the used tool. Here, for example, the Creativity Support Index (CSI) [14] is used. The CSI can be used for different systems or interfaces, covering a wide range of creativity tools. The quality of the tool is measured by six factors: Results Worth Effort, Expressiveness, Exploration, Immersion, Enjoyment, and Collaboration.

\section{Creativity and Cognitive Load}

Nguyen and Zeng [34] deduced that mental stress and creativity correlate in a nonlinear way, as there is a sweet spot for mental stress that should neither be surpassed nor undercut to support creativity in a similar way to the Yerkes-Dodson Law [48]. As mental stress is highly dependent on both the perceived mental workload and mental capacity of the user, it is reasonable to design creative tools as such, that the user's mental workload is kept within a healthy frame to support his or her creativity. Within the research fields of HumanMachine and Human-Computer Interaction, multimodal and human-centered design approaches were found to be able to free up mental resources of the user and therefore decrease his or her perceived mental workload [35]. To achieve this, cognitive models such as the theory of multiple ressources by Wickens [44] can be considered to prevent overstressing of cognitive resources. Nevertheless, creativity supporting
Table 1. A selection of different methods for measuring creativity in humans

\begin{tabular}{ll}
\hline Area & Test \\
\hline Perception and Imagination & Rorschach [38] \\
& Thematic Apperception Test \\
& {$[32]$} \\
Attitude and Personality & NEO-FFI (Openness for Ex- \\
& perience) [18] \\
Drawing & Figural Test in Torrance \\
& Tests of Creative Thinking \\
& [42] \\
Performance & Torrance Tests of Creative \\
& Thinking [42] \\
& Berlin Intelligence Structure \\
& Test [26] \\
\hline
\end{tabular}

tools should also be challenging and interesting to use to avoid undercutting the productive sweet spot for the mental stress of the creator.

\section{Creativity and Wellbeing}

In addition to the relations between mental stress and creativity, relations between wellbeing and creativity can also be found. Thus, people who exercise creative professions show a higher subjective wellbeing [21]. This is reflected in higher mean values in life satisfaction, worthwhileness, and happiness. Not only creative professionals profit from the positive influences of creativity. These effects affect both younger and older people. The Creativity and Aging Study [17] examined the influence of creativity on the health and wellbeing of older people. It was found that by participating in cultural programs led by artists, older people were able to achieve improvements in general and mental health, overall functioning and wellbeing [17]. Rose and Lonsdale [39] came to similar conclusions. In their study they showed that landscape painting can improve the subjective well-being of older people. In the work of [37] visual art-making was helpful to enrich the mental life of older women. Similar results can also be found for young people. Burnard and Dragovic [11] describe that collaborative creativity in music instrument classes improves pupils' wellbeing. In all these studies, the authors cite the sense of autonomy and experience of the participants as competent, active individuals as important points promoted by creative tasks.

\section{Related Work in Multimodal (Pen) Input}

The benefits of using multi-touch and pen input in tandem on large interactive surfaces, such as tabletops are well explored (e.g., [9, 20, 24, 30, 45, 49]). However, with small-sized interactive surfaces, such as with tablets bimanual touch input faces more constraints. For example, in addition to screen sizes being insufficient, it is easier to cause undesired screen occlusion. Yoon et al. [49] have studied how pen and touch can be used in combination to compensate insufficient space on a tablet device for making annotations by tearing the digital paper and creating additional space. An approach to overcome interaction constraints with uni-modal solutions without introducing additional user interaction steps is the integration 
of alternative input modalities. To this end, researchers (e.g., $[6,12])$ have suggested to make use of visual and interactive space around mobile devices. This trend of expanding the interaction space of touch screen devices, such as mobiles and tablets, above and around the actual touch-sensitive screens has been adopted consequently by many researchers (e.g., [23, $33,47,50])$. The expansion shows promise to address many of the limitations in unimodal touch interaction with interactive screens/surfaces by supporting alternative input techniques and combining them with touch input.

Combinations of Mid-air and touch input have also been proposed for various other devices and contexts, such as automotive [5] and retail [7], addressing typical performance issues with small-sized screens and occlusion. Chen et al. [15], for example, suggested to combine pre-touch or post-touch gestures with touch allowing alternative forms to perform operations, such as to zoom by tabbing the screen followed by circling with the finger above the screen. Hinckley et al. [23] suggested to adapt touch interfaces depending on the posture of the approaching hand, for example to differentiate a two-fingerzoom intention from a thumb-tab intention on the screen and thus reduce information overload on the screen. In contrast Aslan et al. [1] have explored the user experience of pre-touch proxemic interactions with touch targets. They (e.g., [4, 8, 2]) have also demonstrated users' interest in (i) pre-touch proxemic input and (ii) combinations of mid-air input and pen input. Others (e.g., [3, 12, 36, 25]) have presented functional prototypes demonstrating benefits of bimanual touch input combined with another input modality. Thus, the general design space of pen+mid-air gestures (e.g., $[3,8]$ ) has already been studied, including literature reviews and contextual inquiries to improve the understanding of relevant pen-based operations.

While previous research has shown benefits of combining midair and touch or pen considering contextual task performance and user experience, so far the interaction space's ability to support creativity has not been studied. We believe that as a bodily and bimanual interaction technique, pen and midair interaction has the potential to foster creativity support by, for example enabling expressive and enjoyable interactions [27].

\section{PROTOTYPE FOR MULTIMODAL PEN-BASED DRAWING}

It is undeniable that digitalization is changing the nature of tools and materials, which are used in artistic practices in professional and non-professional settings. For example, today it is common that even young children start to express their ideas and explore their creativity by drawing on tablets as digital canvases. In fact, how we draw on screens has been inspired for a long time by how we draw on paper; and, users' imagination is often still "constrained" by the materiality of the original physical drawing materials and the original form of contact-based pen and paper interaction. Thus, there are many software-based tools, which resemble traditional tools, such as various forms of virtual brushes, erasers, etc. However, one of the interaction design issues with digital pens seems that while they are physical tools and may look and feel similar to a"static" analog pen their key attribute is that they are "malleable" and can offer and represent multiple tools. For example, while a digital pen can paint with any digital color the physical pen (i.e., the physical representation of the digital pen) itself often lack any means to directly signal or represent its current digital configuration or mode. Instead, the canvas itself is often used indirectly to indicate the mode of the digital pen. We believe that such a potential mapping issues can be addressed by considering what has also been coined as the "turn to materiality"; i.e., a stance towards interaction design which advocates sensitivity towards materials and carefulness in composing hard- and software for physical interfaces [43].
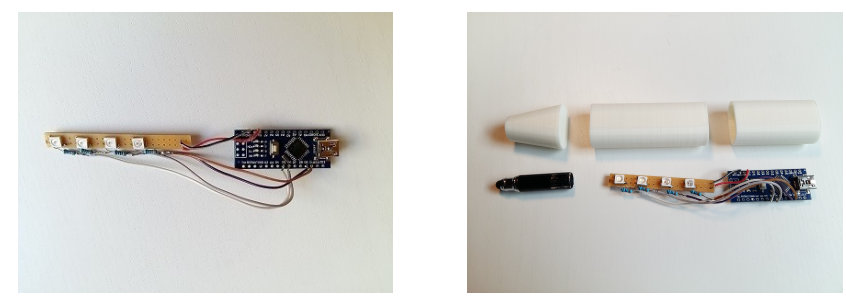

Figure 1. The First version of the pen augmented with a display.

Figure 1 presents a first prototype of a digital pen, which would be able to directly signal and represent its its digital configuration. This first pen prototype was a capacitive pen, which integrated the Arduino Nano into its body. However, we found that the touch accuracy of this capacitive pen was nonsatisfactory, not really state of the art anymore, and thus less performant compared to, for example, the Microsoft Surface's pen. Furthermore, we considered the size of this first pen prototype as too big. Therefore, we decided to create a second prototype, which is essentially not a standalone pen but an extension for the surface pen, allowing us to combine the surface pen's accuracy with the first pen prototype's capability to display user feedback. We have also separated the Arduino Nano from the pen to decrease the weight of the pen. In the first prototype we used four SMT 5050 RGB LEDs and the in the second we used three, because in our tests we received adequate display results with already 3 LEDs.

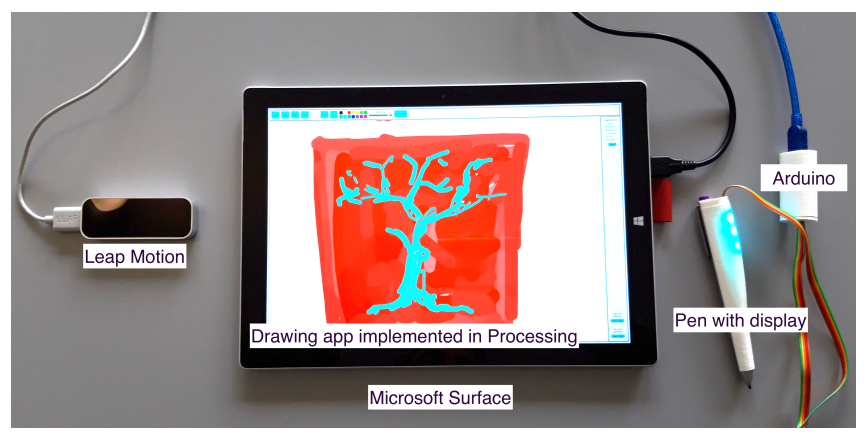

Figure 2. Overview of the setup of the final prototype.

The second and final iteration of the pen prototype is depicted in Figure 2 as part of the complete interaction setup, which consisted of the pen, a Leap Motion sensor (i.e. a 3D sensor, which is able to sense user's hands above the sensor), and a drawing application, which we developed to demonstrate the potential of the multimodal setup. The different hardware parts are connected via USB on the Surface device utilizing a 
USB hub. The Arduino receives commands (over a serial port) from the drawing application to set the LEDs to a desired color range. The Leap Motion sensor recognizes the distance of the second hand to the leap motion and sends distance information to the drawing application. Depending on the set mode (i.e., multimodal on or off) on the drawing application this distance data is mapped to parameters of functions selected in the drawing application. In a nutshell, the 3D sensor provides data similar to a slider but allows more flexibility in terms of where to place the sensor without requiring screen space or potentially causing occlusion issues. We decided to use a 3D sensor, because related work (e.g., $[1,27])$ suggests that pen and mid-air input is associated with increasing expressivity ,and thus potentially fosters creativity.

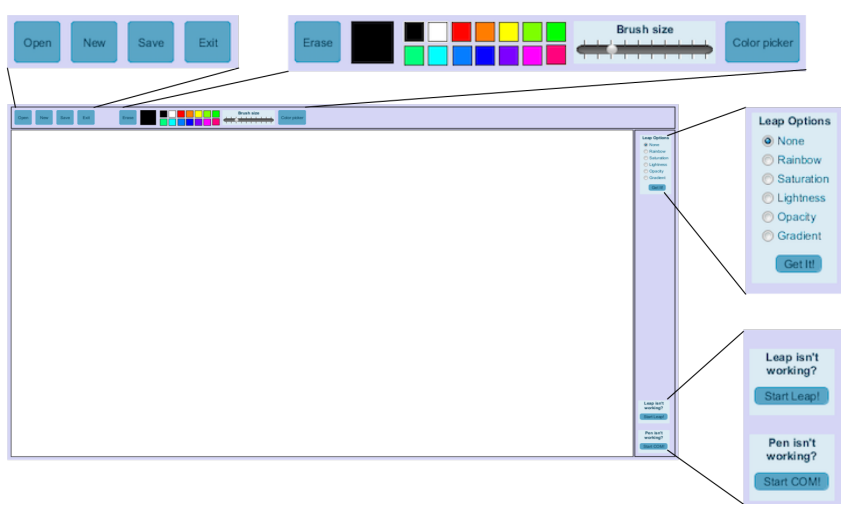

Figure 3. Overview of GUI of the drawing app.

The drawing application (see Figure 3) provides a widget with check boxes to control how distance data is mapped to functions which can be selected on the user interface. We implemented five exemplary target functions (i.e., color opacity, color saturation, color lightness, color from a rainbow range, color from a gradient range, and brush size) and combinations of the functions. For example, when a user selects the gradient function a color picker window pops up where the user can pick two colors and afterwards draw within the range of this two colors by adjust their second hands distance to the 3D sensor (also while drawing). Figure 4 provides exemplary brush strokes.

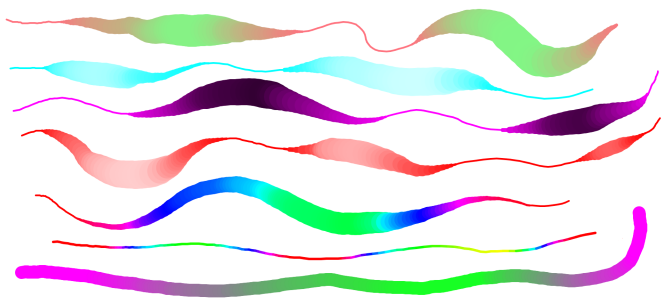

Figure 4. Exemplary strokes of the multimodal pen prototype.

\section{USER STUDY}

In order to explore the influence of using the multimodal pen prototype on creativity support we conducted a study with 26 participants. Our hypothesis was that the multimodal
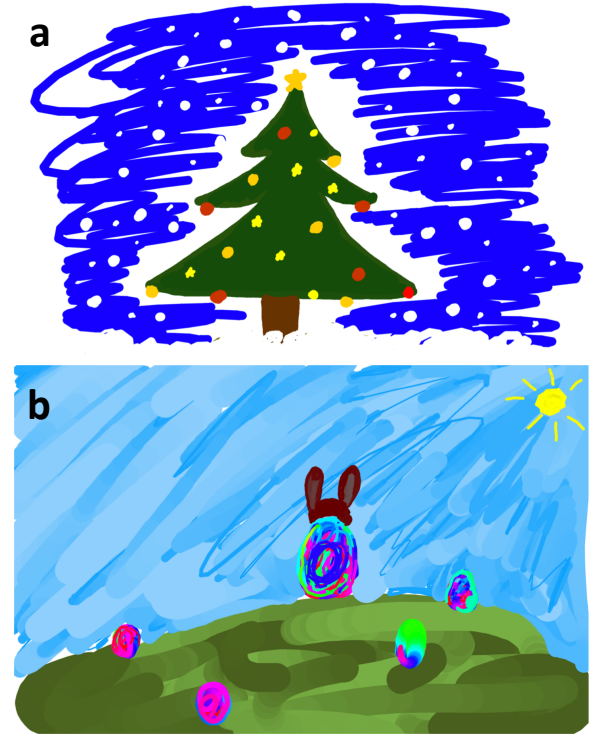

Figure 5. A participants post card designs, which were created in a) the baseline condition, and b) the experimental condition.

prototype would be perceived as supporting creativity more than a baseline version.

The baseline version would not offer bimanual input, not use the pen's integrated display, and would not offer the compound functions (e.g., change brush size while drawing, or change color attributes while drawing). Thus, the aim of the user study was to test if the "multimodality" of our exemplary interaction design would cause perceived support of creativity and we wanted to shed some light into reasons for why that may be the case.

\section{Procedure}

We conducted a within-subjects study, using counterbalancing to reduce any bias due to order. We recruited a heterogenous group of participants of which 11 reported to be female and 15 male. The mean age of the participants was 35 . In summary, five participants reporting to be between 56-65, two between 46-55, three between 36-45, nine between 26-35, and seven younger than 26. Many participants were recruited from outside the university. Half of the participants reported to have no experience using digital pens, while the rest reported to have at least some experience using digital pens. Two participants were left handed. Participants were also asked to rate their own creativity skills on a five-point Likert scale.

In order to measure creativity support itself, we used the Creativity Support Index (CSI) questionnaire [16], which measures six (weighted) dimensions of creativity support and providing a CSI score. This questionnaire is inspired by the NASA TLX questionnaire [22], which is well known in the multimodal interaction community. Similar to the NASA TLX questionnaire participants are asked to weight each dimensions' value for the specific task that they are asked to complete (using a Paired-Factor Comparison Test). One of the six dimensions relates to collaboration and only applies to collaborative tasks and can be left out, which we did. 
Average weight for each dimension of creativity support for the post card design task

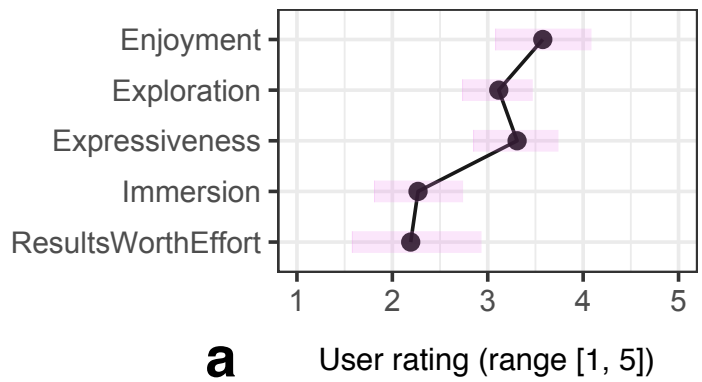

Average score reported for each dimension of creativity support for both conditions

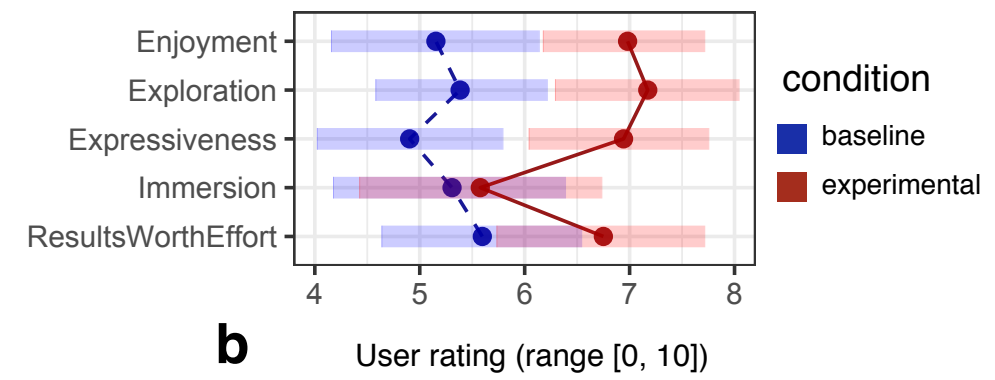

Figure 6. Overview of results considering the five dimensions of creativity support measured by creativity support index questionnaire. Error bars denote $95 \%$ confidence intervals.

The task we asked participants to complete was to design a post card. In order to reduce a learning bias we asked them to design post cards for two different events (i.e., a Christmas post card and a post card for Easter) in counterbalanced order over participants and modalities. Figure 5 provides two designs of one participant as an example.

Consequently, each participant created two post card designs. For each design they had 10 minutes. Participants were asked to fill out the CSI questionnaire (on a separate notebook) after each task. Participants were also asked to "think aloud" whenever possible. At the end a semi structured interview was conducted with each participant. Welcoming each participant and providing them the minimal necessary introduction (written on paper) to the study, including formalities and task took about 10 minutes. Overall, we spend about 50 minutes with each participant conducting the study.

\section{Results}

In the following, we first present the results of the CSI questionnaire, which can be used to answer if participants perceived a significant difference in creativity support between the two conditions they were asked to use. Afterwards we provide results of a qualitative analysis, which focusses on our observations during the tasks and the data collected in the semi-structured interviews.

\section{CSI Index}

Figure 6 depicts results considering the five dimensions of creativity support that the CSI questionnaire measures. Figure 6a shows how participants weighted each of the dimensions considering the post card design task. They have rated Enjoyment, Exploration, and Expresivness as more "important" than the dimensions Immersion and ResultsWorthEffort, which seems understandable considering the playful nature of the task and potentially positive associations with the holidays.

Figure 6b depicts participants' ratings of each of the five dimensions of creativity support for both interaction conditions (i.e., baseline and experimental/multimodal). Since our hypothesis was that the experimental condition would support creativity more than the baseline version we conducted onetailed paired T-tests to compare the user ratings for both conditions. As expected the experimental condition received signifi- cantly higher user ratings considering Enjoyment $(\mathrm{t}=3.56, \mathrm{p}$ $<.001, \mathrm{r}=.58)$, Exploration $(\mathrm{t}=2.83, \mathrm{p}<.001, \mathrm{r}=.49)$, Expresivness $(\mathrm{t}=4.29, \mathrm{p}<.001, \mathrm{r}=.65)$, and ResultsWorthEffort $(\mathrm{t}=1.74$, $\mathrm{p}=.047, \mathrm{r}=.33)$. In contrast the difference in the ratings for Immersion $(\mathrm{t}=.4, \mathrm{p}=.35, \mathrm{r}=.08)$ was non-significant.

As we expected the multimodal pen prototype indeed supports creativity significantly more. In our sample, all dimensions of creativity support but Immersion show significantly higher ratings from participants. Thus, the multimodal pen prototype also achieved a significantly higher CSI Score (see Figure 7) $(\mathrm{t}=2.812, \mathrm{p}<.001, \mathrm{r}=.49)$ compared to the baseline condition.

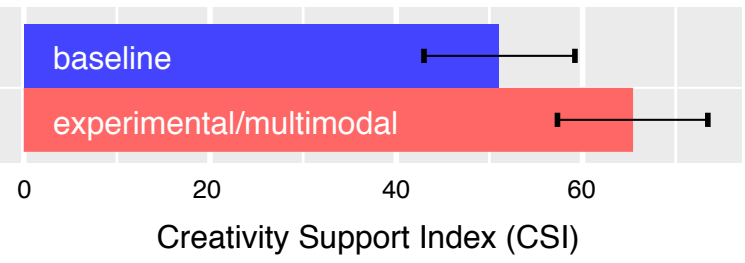

Figure 7. Creativity Support Index scores for both conditions

\section{Participant Feedback}

With each participant, a semi-structured interview was conducted after they had finished designing both post-cards. Our intention was to identify potential reasons for why they may have liked or disliked aspects of the multimodal condition and how it may have influenced their perception of the interaction technique supporting creativity. Questions we asked were for example, Is there something specific that you liked in the "multimodal" design? and Is there something specific that you disliked in the "multimodal" design? followed by Why questions to understand participants individual reasons.

Figure 8 depicts some results of the additional questions, considering how helpful participants found the Leap Motion and the LEDs display on the pen as parts of the interaction design. Mean ratings are above the average score of three, and thus, it seems that participants leaned towards agreeing that these multimodal aspects are helpful. Similarly, they seem also to lean towards agreeing that the multimodal pen prototype would support their creativity on the long run if they used it regularly. In addition, we asked participants to rate on a five point Likert 
scale "ease of use" of the experimental/multimodal $(\mathrm{M}=4.0$, $\mathrm{SD}=1.12)$ and the baseline condition $(\mathrm{M}=3.9, \mathrm{SD}=1.0)$ with both modalities receiving similarly positive ratings.

\section{"Leap motion was helpful."}

\section{"LEDs display on the pen was helpful."}

"Experimental condition would support my creativity if I would use it regularly."

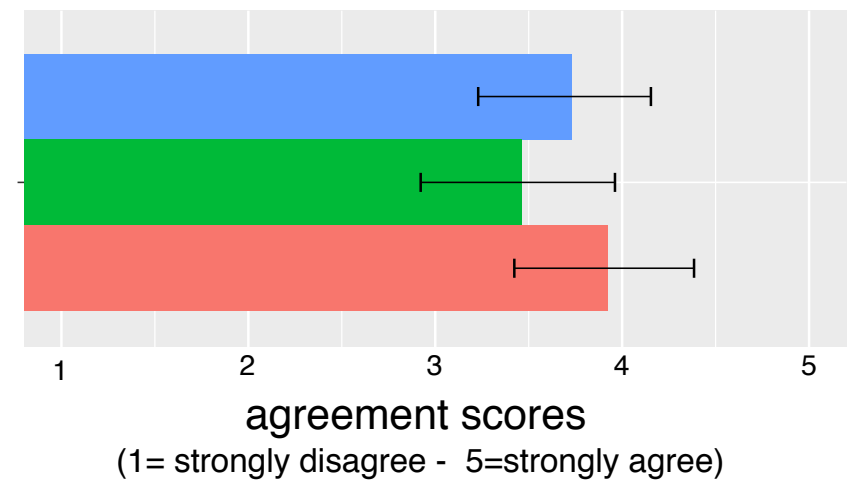

Figure 8. Participants ratings about the helpfulness of multimodality and long-term expectations considering creativity support.

Furthermore, nine participants mentioned the LEDs display on the pen as the key features that they liked about the pen. Participants stated as reasons, for example, "The color is visible before one starts to draw", "The fact that the current color is visible on the pen", and "There is no need to use multiple different colored pens", "[I liked the] color display when using the Rainbow effect", and simply "the light effects'. Eleven participants mentioned performance and ergonomics related reasons, including "Good haptics and easy to use", "Good reaction times", "That it works fast", "Pen is very light, it fits comfortably in your hand, barely noticeable", "The precise tracking and the additional sensor [Leap Motion]". Two participants explicitly mentioned as the best feature being able to manipulate the stroke size while drawing and another two referred in general to duality e.g., "Parallel control over effects while drawing". One could argue that participants seem to have explicitly perceived the potential of the multimodal techniques to improve pragmatic and hedonic qualities.

We also asked participants for both drawing conditions if they would have liked to keep designing (beyond the 10 minutes slot that they were given) and why they would have liked to continue in case they answered yes. While only three participants answered yes for the baseline condition twenty-two participants answers yes for the experimental/multimodal condition. One participant stated " [because] $i$ forgot the time. I was blown away". Another participant stated " [because] the usage of the left hand with the system is intuitive after a few minutes. One is drawn into the task and a joy for drawing develops". Indeed, nine participants explicitly mentioned that they would have liked to keep drawing because they enjoyed it and thought that it was fun. Four participants mentioned they would have liked to keep exploring the features/effects in more detail. Participants who stated that they would have (also) liked to keep drawing in the baseline condition stated as reasons "[because] the pen reacted good in itself" and another stated "Because I never used a digital pen before".

When we asked participants to tell us what they didn't like with the multimodal interaction technique their critique often related to the drawing application not offering all the functions "professional" drawing application would provide, such as a function to draw straight lines and shapes, or difficulties to draw dots. Some also mentioned that the graphical user interface was not very usable, because check boxes were too small (which were the same size in both conditions). One participant criticized that the design only allowed distance to be used for two-handed manipulation. They wished to be able to move their hands sideways to manipulate additional functions. Only, two participants mentioned that the performance was not accurate or good enough.

The last question we asked was "What would need to be changed in the interaction technique(s) to support creativity". Participants answers were divers. Many participants proposed to add additional functionality. For example, one participant mentioned adding pressure sensitivity and another participant would want to have more colors (e.g., neon and glitter) and the ability to integrate camera photos. Another participant simply wished an undo function. It was also suggested to use Bluetooth instead of the usb cable connection. Four participants had suggestions related to personalizing the leap motion's "sensitivity" (i.e., how much one would have to move one's hand to access the whole color/size range).

\section{Thematic analysis}

In order to systematically analyze all the observations and participants' utterances made during the study we conducted a thematic analysis [10]. To this end, we transcribed relevant comments (e.g., direct references to creativity or to the interaction technique) and added them to the data collected through the post-hoc interviews. Then words and sentences that occurred repeatedly or were explicitly mentioned as important by participants were marked and included for each condition into an extensive mind map, which then were diverged into three common descriptors (e.g., by merging similar branches in the mind-map) to reduce redundant information in the mindmaps and to identify main themes for each condition. Figure 9 provides for both conditions the main themes, which capture the essence of what participants described.

The themes that came up when participants spoke about the baseline condition were handling, conventional and standard tasks. Participants argued that the baseline condition mapped to a standard way of interacting with a pen. They considered it as method to solve standard tasks, offering a conventional handling very similar to traditional pen and paper. One participant (f, 58) who criticized the experimental condition explicitly stated "I am old-fashioned and I still like to paint with colored pencils". Indeed, we observed that participants age seemed to be important considering their perception of the experimental design's ability to support creativity.

We computed Pearson's $r$ to shed light into possible significant relations between participants' age and their ratings consid- 

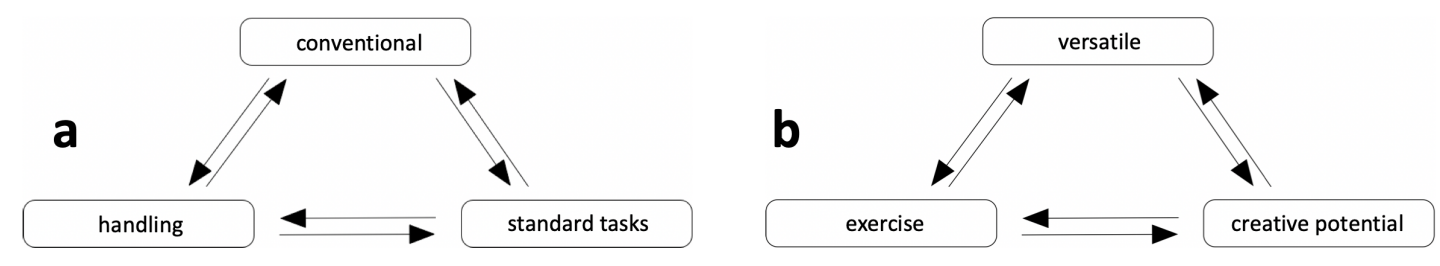

Figure 9. Results of the thematic analysis for a) the baseline condition and b) the experimental/multimodal condition.

ering the five dimensions of creativity support. We found a significant correlation between age and ratings for Exploration $(\mathrm{r}=-.42, \mathrm{n}=26, \mathrm{p}=.03)$, and between age and participants' ratings of ease of use of the multimodal pen $(r=-.48, n=26$, $\mathrm{p}=.013$ ). Consequently, with increasing age users are less likely to consider the multimodal pen design as supporting their creativity and more accurately Exploration as a dimension of creativity support. In a nutshell, older participants felt less motivated by the multimodal design to explore new ideas and techniques.

The themes that emerged about the experimental condition were versatile, exercise and creative potential. Participants seem convinced that the versatile character of the multimodal interaction technique, which allowed them to be flexible/versatile provided them a creative potential which they believed could be achieved/fused through exercise. Exercise refers to participants considering the use of both hands and the display on the pen as something new , and that exercise would provide them more benefits over time. Versatile referred to the multimodality and how it provided alternatives which no other drawing app provided and which was non-standard.

In summary, participants feedback highlights that both conditions were usable. But only the experimental/multimodal condition was associated with a potential to increase/support "their" creativity by both motivating exercise and versatility. Participants have also associated the multimodal technique with more positive emotions, such as fun or "flow" (e.g., loss of conscious perception of time), which could be part of participants willingness for exercise and trying out new approaches/ideas.

\section{DISCUSSION}

Drawing is a ubiquitous activity, which we as humans learn to perform already in our childhood years and which help us to express ourselves, stimulate our thoughts, and engage intriguing ideas. For some, this playful activity may turn into a profession or a passion. As adults we may become aware of how we are empowered by the tools and materials we have at our disposal and explore new (and dgital) options.

In this paper, we have so far argued and demonstrated that (multimodal) technology has the potential to support creativity in drawing/sketching activities. In the following, we will discuss the research results presented in this paper considering insights we have from taking part in sketching communities in our town and observing and interviewing members from these communities. Our intention is to describe the validity of creativity supporting technology for exemplary communities. Specific to these communities is their members' appreciation for sketching as a skill/craft and a desire to improve their related competences. We concretely draw insights from two (intersecting) groups that regularly meet for urban or nude sketching. They meet for the benefit of social contact and moreover consider these meetings as an opportunity to learn new techniques from others, exercise, and keep their skills sharp for their professional daytime work as, for example gold smiths, layouters, concept artists, graphics designer, or woodcarvers. Both groups focus on the skill to sketch what they see in 3D strictly without relying on any 2D representations, such as photography. Each community meets typically once a week with 10-15 members attending sessions. Some members attend meetings only once in two weeks or a month, thus attendees of these meetings vary with a core group of about five member who "always" attend meetings. The insights we present are based on one researcher being a long time member of both groups, a second researcher attending the nude sketching community for three weeks, and a third researcher interviewing the urban sketchers on two occasions.

\section{Attitudes Towards Technology}

One may think that communities, such as the urban sketchers who meet to draw urban places or the nude sketchers who meet to draw nudes are not receptive to technology. Indeed, people who occupy an office job will sometimes cherish natural and conventional materials and welcome the challenge of using water-colors and sketchbooks where an undo is not a real option. But overall members' attitudes towards technology and tech-adoption is very divers with actually most members being positive towards technology's potential to improve the outcome of their sketching activities. They tend to be willing to discuss technology, and how it may help or hinder their personal development. Both, limitations and potentials of technology are often topic of discussions. For example, group members will argue against using technology because of reflective screens and loss in haptic qualities when sketching on screen. On the one side, the lack of "materiality" will be criticizes. On the other side, they will motivate using technology because of "flexibility" (e.g., number of features or brushes) and increasing portability of modern devices.

The actual tools that are used by members of these groups for sketching vary. Some members use only conventional pens and papers, but increasingly more members seem to start drawing on tablets. At the time, the work at hand is conducted iPad-Pro's reactivity and "high-end" painting capabilities is often referred to as benchmark whenever someone introduces or demonstrated another tablet in these meetings. For example, once a long-time member brought a new tablet, which uses e-ink technology (i.e., the Remarkable tablet), which he intro- 
duced to the other members, stressing out how this tablet was not like the iPad, it was good for using outside, especially on sunny days. He also stressed out how it was very responsive, and how despite only allowing black and white sketches he valued it since he could still color his (vector graphics) sketch later on his personal computer. His argument was that the iPad is great but he really doesn't need a complete computer for his sketching activities. When we asked two members who were appreciating the capabilities of the iPad Pros for why they didn't use an iPad, they said they simple can't afford it. One of these two had an Android based tablet which she occasionally used. Overall, the majority of members has already adopted technology in one way or another way. Most of them upload their drawings to their computers to share them with others or to continue working on them in digital format on their personal computers.

\section{Age and Creativity Support}

Interestingly, the ages of the members of sketching communities are divers. Many of our discussions about using technology and what technology should be used has been conducted with older members, who may have been drawing for decades on pen and paper and seem very intrigued by (but not completely satisfied by) the opportunities technology is starting to offer. Indeed there seems to exist both a need and an extra challenge for older adults, which we have also observed in the user study. Especially for older people who might start to deal with a decrease in physical ability (e.g., decrease of sight and motor control) multimodal technology that may not only compensate decrease in cognitive ability but allow them to make use and increase their already existing competences through offering new doable opportunities. However, we have also came to understand that with older adults we, as researchers and designers might be more careful in our multimodal design explorations, since the "sweet spot" on the range between cognitively challenging and exhausting will vary compared to, for example, their younger selves. And of course it would also be "good" if we were able to provide "artists" not only new multimodal ways to express themselves but also long term adaptive solutions, which may adapt to their changing skills, competences, and cognitive abilities over time. In our own future work we aim to explore reinforcement learning methods to further address this potential of (multimodal) techniques. Because, such solutions would allow them to continue an activity that many people in the sketching communities see as a key aspect of their (social) life and consequently psychological well-being. We have also highlighted the important relationship between creativity support, wellbeing and aging in the background sections. However, it is important that the aim is to flourish people's crafts and skills and not replacing them (or trying to replace it) with completely autonomous solution, which would have no value for the communities that we observed.

\section{"Traditional” Creativity Support Practices}

It should not be surprising that sketching communities already apply some "creativity support" techniques to break out of usual routines and help to build new skills. For instance, it is common for nude sketchers to limit a drawing session's time frame to only a few minutes (and some even use their not preferred hand) to draw. Most techniques are easy ways to increase the level of (cognitive) challenge and help members to increase their sketching competence or "force" them to try out new techniques (e.g., one line drawings).

The user study results have clearly presented why participants have perceived the multimodal pen as significantly more supporting their creativity. They felt significantly more Enjoyment, Exploration, Expressivenes, and ResultsWorthEffort. We did not design the multimodal pen prototype with the intention to be a replacement or imitation of the digital pen, but rather offer new and additional modalities for input while drawing. Consequently, participants had more opportunities to challenge themselves in case they felt the "baseline options" were not demanding enough. However, there was still a risk that the additional input techniques could have been dismissed or even could have interfered with participants' creativity, since constraining design activities can support creativity by providing friction for creativity [28].

Overall, we believe that providing alternative interaction modalities to existing tools will have the potential to increase creativity support. There is especially the potential to enable "artists" to "bend" new modalities towards their own needs to challenge themselves at the right level and to continuously foster their skill levels and competences. We may require performing more and deeper ethnomethodological inquiries with users in the field and communities such as the urban and nude sketchers to shed light on how new techniques will result in new creativity practices. Nevertheless, we hope our research so far will inspire fellow researcher to join the study of how multimodal interaction can support creativity.

\section{CONCLUSION}

We believe that creativity as a cognitive skill is highly relevant in and for the design of multimodal interfaces. Thus, a general aim of our research was to shed light on the potential of multimodality to support creativity and consequently to also inspire fellow researchers to explore how their research in multimodal interaction may also support creativity. In order to demonstrate how a multimodal design can support creativity we have first presented a multimodal (digital) pen prototype and reported in detail on a comparative user study with 26 participants comparing the prototype to a baseline version (i.e., a digital pen without the multimodal features). Results have highlighted significant differences, with the multimodal pen receiving higher Creativity Support Index (CSI) scores and supporting significantly more Enjoyment, Exploration, Expressiveness, and ResultsWorthEffort as dimensions of creativity support. We complemented the results based on the CSI questionnaire with results of a thematic analysis and insights we gathered from "applied ethnographies" with two sketching communities in our town. We hope that the research at hand will also contribute and inspire contributions to a larger agenda of designing technology to support human flourishing.

\section{ACKNOWLEDGMENTS}

blinded for review 


\section{REFERENCES}

1. Ilhan Aslan and Elisabeth André. 2017. Pre-touch Proxemics: Moving the Design Space of Touch Targets from Still Graphics Towards Proxemic Behaviors. In Proceedings of the 19th ACM International Conference on Multimodal Interaction (ICMI 2017). ACM, New York, NY, USA, 101-109. DOI : http://dx.doi.org/10.1145/3136755.3136808

2. Ilhan Aslan, Björn Bittner, Florian Müller, and Elisabeth André. 2018. Exploring the User Experience of Proxemic Hand and Pen Input Above and Aside a Drawing Screen. In Proceedings of the 17th International Conference on Mobile and Ubiquitous Multimedia (MUM 2018). ACM, New York, NY, USA, 183-192. DOI : http://dx.doi.org/10.1145/3282894.3282906

3. Ilhan Aslan, Ida Buchwald, Philipp Koytek, and Elisabeth André. 2016a. Pen + Mid-Air: An Exploration of Mid-Air Gestures to Complement Pen Input on Tablets. In Proceedings of the 9th Nordic Conference on Human-Computer Interaction (NordiCHI'16). ACM, New York, NY, USA, Article 1, 10 pages. DOI: http://dx.doi.org/10.1145/2971485.2971511

4. Ilhan Aslan, Julian Kraus, and Elisabeth André. 2016b. LeapArm-Facilitating Sensory Spaces for Mid-air Gestural Interaction. In Proceedings of the 9th Nordic Conference on Human-Computer Interaction (NordiCHI '16). ACM, New York, NY, USA, Article 106, 6 pages. DOI:http://dx.doi.org/10.1145/2971485.2996741

5. Ilhan Aslan, Alina Krischkowsky, Alexander Meschtscherjakov, Martin Wuchse, and Manfred Tscheligi. 2015a. A Leap for Touch: Proximity Sensitive Touch Targets in Cars. In Proceedings of the 7th International Conference on Automotive User Interfaces and Interactive Vehicular Applications (AutomotiveUI '15). ACM, New York, NY, USA, 39-46. DOI : http://dx.doi.org/10.1145/2799250.2799273

6. Ilhan Aslan and Antonio Krüger. 2004. The Bum Bag Navigator (BBN): an advanced pedestrian navigation system. AIMS 2004 (2004).

7. Ilhan Aslan, Thomas Meneweger, Verena Fuchsberger, and Manfred Tscheligi. 2015b. Sharing Touch Interfaces: Proximity-Sensitive Touch Targets for Tablet-Mediated Collaboration. In Proceedings of the 2015 ACM on International Conference on Multimodal Interaction (ICMI '15). ACM, New York, NY, USA, 279-286. DOI : http://dx.doi.org/10.1145/2818346.2820740

8. Ilhan Aslan, Tabea Schmidt, Jens Woehrle, Lukas Vogel, and Elisabeth André. 2018. Pen + Mid-Air Gestures: Eliciting Contextual Gestures. In Proceedings of the 20th ACM International Conference on Multimodal Interaction (ICMI '18). ACM, New York, NY, USA, 135-144. DOI : http://dx.doi.org/10.1145/3242969.3242979

9. Peter Brandl, Clifton Forlines, Daniel Wigdor, Michael Haller, and Chia Shen. 2008. Combining and Measuring the Benefits of Bimanual Pen and Direct-touch
Interaction on Horizontal Interfaces. In Proceedings of the Working Conference on Advanced Visual Interfaces (AVI '08). ACM, New York, NY, USA, 154-161. DOI : http://dx. doi .org/10.1145/1385569.1385595

10. Virginia Braun and Victoria Clarke. 2006. Using thematic analysis in psychology. Qualitative Research in Psychology 3, 2 (2006), 77-101. DOI: http://dx.doi.org/10.1191/1478088706qp063oa

11. Pamela Burnard and Tatjana Dragovic. 2015. Collaborative creativity in instrumental group music learning as a site for enhancing pupil wellbeing. Cambridge Journal of Education 45, 3 (2015), 371-392. DOI: http://dx.doi.org/10.1080/0305764X.2014.934204

12. Alex Butler, Shahram Izadi, and Steve Hodges. 2008. SideSight: Multi-"Touch" Interaction Around Small Devices. In Proceedings of the 21 st Annual ACM Symposium on User Interface Software and Technology (UIST '08). ACM, New York, NY, USA, 201-204. DOI : http://dx. doi .org/10.1145/1449715.1449746

13. Rafael A Calvo and Dorian Peters. 2014. Positive computing: technology for wellbeing and human potential. MIT Press.

14. Erin A Carroll, Celine Latulipe, Richard Fung, and Michael Terry. 2009. Creativity factor evaluation: towards a standardized survey metric for creativity support. In Proceedings of the seventh ACM conference on Creativity and cognition. ACM, 127-136.

15. Xiang 'Anthony' Chen, Julia Schwarz, Chris Harrison, Jennifer Mankoff, and Scott E. Hudson. 2014. Air+Touch: Interweaving Touch \&\#38; In-air Gestures. In Proceedings of the 27th Annual ACM Symposium on User Interface Software and Technology (UIST '14). ACM, New York, NY, USA, 519-525. DOI: http://dx.doi.org/10.1145/2642918.2647392

16. Erin Cherry and Celine Latulipe. 2014. Quantifying the creativity support of digital tools through the creativity support index. ACM Transactions on Computer-Human Interaction (TOCHI) 21, 4 (2014), 21.

17. Gene D Cohen, Susan Perlstein, Jeff Chapline, Jeanne Kelly, Kimberly M Firth, and Samuel Simmens. 2006. The impact of professionally conducted cultural programs on the physical health, mental health, and social functioning of older adults. The Gerontologist 46, 6 (2006), 726-734.

18. PT Costa, RR McCrae, and NEO Revised. 1992. Personality Inventory and NEO Five-Factor Inventory: Professional Manual. FL: Psychological Assessment Resources (1992).

19. Umer Farooq, John M Carroll, and Craig H Ganoe. 2005. Supporting creativity in distributed scientific communities. In Proceedings of the 2005 international ACM SIGGROUP conference on Supporting group work. ACM, 217-226. DOI :

http://dx.doi.org/10.1145/1099203.1099242 
20. Mathias Frisch, Ricardo Langner, and Raimund Dachselt. 2011. Neat: A Set of Flexible Tools and Gestures for Layout Tasks on Interactive Displays. In Proceedings of the ACM International Conference on Interactive Tabletops and Surfaces (ITS '11). ACM, New York, NY, USA, 1-10. DOI :

http://dx.doi.org/10.1145/2076354.2076356

21. Daniel Fujiwara, Paul Dolan, and Ricky Lawton. 2015. Creative occupations and subjective wellbeing. Nesta: London, UK (2015).

22. Sandra G Hart and Lowell E Staveland. 1988. Development of NASA-TLX (Task Load Index): Results of empirical and theoretical research. In Advances in psychology. Vol. 52. Elsevier, 139-183.

23. Ken Hinckley, Seongkook Heo, Michel Pahud, Christian Holz, Hrvoje Benko, Abigail Sellen, Richard Banks, Kenton O'Hara, Gavin Smyth, and William Buxton. 2016. Pre-Touch Sensing for Mobile Interaction. In Proceedings of the 2016 CHI Conference on Human Factors in Computing Systems (CHI'16). ACM, New York, NY, USA, 2869-2881. DOI : http://dx.doi.org/10.1145/2858036.2858095

24. Ken Hinckley, Koji Yatani, Michel Pahud, Nicole Coddington, Jenny Rodenhouse, Andy Wilson, Hrvoje Benko, and Bill Buxton. Pen + Touch $=$ New Tools. In Proc. of UIST' 10. ACM, 10.

25. Sungjae Hwang, Andrea Bianchi, Myungwook Ahn, and Kwangyun Wohn. 2013. MagPen: Magnetically Driven Pen Interactions on and Around Conventional Smartphones. In Proceedings of the 15th International Conference on Human-computer Interaction with Mobile Devices and Services (MobileHCI '13). ACM, New York, NY, USA, 412-415. DOI :

http://dx.doi.org/10.1145/2493190.2493194

26. Adolf Otto Jäger, Heinz-Martin Süß, and André Beauducel. 1997. Berliner Intelligenzstruktur-Test : BIS-Test. Hogrefe, Göttingen. http://ub-madoc.bib.uni-mannheim.de/14578/

27. Scott R. Klemmer, Björn Hartmann, and Leila Takayama. 2006. How Bodies Matter: Five Themes for Interaction Design. In Proc. of (DIS'06). ACM, 10.

28. Jonas Löwgren and Erik Stolterman. 2004. Thoughtful interaction design: A design perspective on information technology. Mit Press.

29. Julian Malins and Graham Grant. 2010. Designing creativity tools to support business innovation. In Proceedings of the 1st DESIRE Network Conference on Creativity and Innovation in Design. Desire Network, 181-188.

30. Fabrice Matulic and Moira C. Norrie. 2013. Pen and Touch Gestural Environment for Document Editing on Interactive Tabletops. In Proceedings of the 2013 ACM International Conference on Interactive Tabletops and Surfaces (ITS '13). ACM, New York, NY, USA, 41-50. DOI : http://dx.doi.org/10.1145/2512349.2512802
31. Barbara Motyl and Stefano Filippi. 2014. Integration of creativity enhancement tools in medical device design process. Procedia Engineering 69 (2014), 1316-1325.

32. Henry Alexander Murray. 1943. Thematic apperception test. (1943).

33. Rajalakshmi Nandakumar, Vikram Iyer, Desney Tan, and Shyamnath Gollakota. 2016. FingerIO: Using Active Sonar for Fine-Grained Finger Tracking. In Proceedings of the 2016 CHI Conference on Human Factors in Computing Systems (CHI '16). ACM, New York, NY, USA, 1515-1525. DOI : http://dx.doi.org/10.1145/2858036.2858580

34. Thanh An Nguyen and Yong Zeng. 2012. A theoretical model of design creativity: Nonlinear design dynamics and mental stress-creativity relation. Journal of Integrated Design and Process Science 16, 3 (2012), 65-88.

35. Sharon Oviatt. 2006. Human-centered Design Meets Cognitive Load Theory: Designing Interfaces That Help People Think. In Proceedings of the 14th ACM International Conference on Multimedia (MM '06). ACM, New York, NY, USA, 871-880. DOI : http://dx.doi.org/10.1145/1180639.1180831 event-place: Santa Barbara, CA, USA.

36. Ken Pfeuffer, Ken Hinckley, Michel Pahud, and Bill Buxton. 2017. Thumb + Pen Interaction on Tablets. In Proceedings of the 2017 CHI Conference on Human Factors in Computing Systems (CHI '17). ACM, New York, NY, USA, 3254-3266. DOI : http://dx. doi .org/10.1145/3025453.3025567

37. Frances Reynolds. 2010. 'Colour and communion': Exploring the influences of visual art-making as a leisure activity on older women's subjective well-being. Journal of Aging Studies 24, 2 (2010), 135-143.

38. Hermann Rorschach. 1942. Psychodiagnostics. (1942).

39. Emma Rose and Stephen Lonsdale. 2016. Painting place: Re-imagining landscapes for older people's subjective wellbeing. Health \& place 40 (2016), 58-65.

40. Paul R Sackett, Filip Lievens, Chad H Van Iddekinge, and Nathan R Kuncel. 2017. Individual differences and their measurement: A review of 100 years of research. Journal of Applied Psychology 102, 3 (2017), 254.

41. Ben Shneiderman. 2007. Creativity support tools: Accelerating discovery and innovation. Commun. ACM 50, 12 (2007), 20-32.

42. E P Torrance. 1974. Norms-technical manual: Torrance Tests of Creative Thinking. (1974).

43. Mikael Wiberg. 2018. The materiality of interaction: Notes on the materials of interaction design. MIT press.

44. Christopher D Wickens. 2002. Multiple resources and performance prediction. Theoretical issues in ergonomics science 3, 2 (2002), 159-177. 
45. Mike Wu, Chia Shen, Kathy Ryall, Clifton Forlines, and Ravin Balakrishnan. 2006. Gesture registration, relaxation, and reuse for multi-point direct-touch surfaces. In Horizontal Interactive Human-Computer Systems, 2006. TableTop 2006. IEEE, 8-pp.

46. Yasuhiro Yamamoto and Kumiyo Nakakoji. 2005. Interaction design of tools for fostering creativity in the early stages of information design. International Journal of Human-Computer Studies 63, 4-5 (2005), 513-535. DOI : http://dx.doi.org/10.1016/j.ijhcs.2005.04.023

47. Xing-Dong Yang, Tovi Grossman, Pourang Irani, and George Fitzmaurice. 2011. TouchCuts and TouchZoom: Enhanced Target Selection for Touch Displays Using Finger Proximity Sensing. In Proceedings of the SIGCHI Conference on Human Factors in Computing Systems (CHI '11). ACM, New York, NY, USA, 2585-2594. DOI : http://dx.doi.org/10.1145/1978942 . 1979319

48. Robert M. Yerkes and John D. Dodson. 1908. The relation of strength of stimulus to rapidity of habit-formation. Journal of Comparative Neurology and Psychology 18, 5 (Nov. 1908), 459-482. DOI :

http://dx.doi.org/10.1002/cne. 920180503

49. Dongwook Yoon, Nicholas Chen, and François Guimbretière. 2013. TextTearing: Opening White Space for Digital Ink Annotation. In Proceedings of the 26th Annual ACM Symposium on User Interface Software and Technology (UIST'13). ACM, New York, NY, USA, 107-112. DOI :

http://dx.doi.org/10.1145/2501988.2502036

50. Chen Zhao, Ke-Yu Chen, Md Tanvir Islam Aumi, Shwetak Patel, and Matthew S. Reynolds. 2014. SideSwipe: Detecting In-air Gestures Around Mobile Devices Using Actual GSM Signal. In Proceedings of the 27th Annual ACM Symposium on User Interface Software and Technology (UIST '14). ACM, New York, NY, USA, 527-534. DOI :

http://dx.doi.org/10.1145/2642918.2647380 